\title{
Spread and adoption of enhanced recovery from elective surgery in the English National Health Service
}

\author{
Michael G. Mythen, MD
}

Received: 4 July 2014/ Accepted: 22 October 2014/Published online: 13 November 2014

(c) The Author(s) 2014. This article is published with open access at Springerlink.com

The document Delivering Enhanced Recovery - Helping Patients to Get Better Sooner After Surgery was published by the UK Department of Health Enhanced Recovery Partnership Programme on March 10, 2010. ${ }^{1}$ The forward, in referring to the UK National Health Service (NHS), says:

"Enhanced recovery is transforming NHS elective and cancer care pathways by using a number of evidence-based interventions as a model of care enabling patients to recover sooner following surgery. This guide, developed using learning from centres across the UK, provides a starting point to support implementation of enhanced recovery."

By November 2013, a progress review entitled Enhanced Recovery Care Pathway reported successful national implementation in four main types of elective surgery, colorectal, gynecological, orthopedic, and urology. ${ }^{2}$ Thousands more surgeries were being performed in 170,000 fewer bed days. Target lengths of stay had been achieved, readmission rates had not increased, and both quality and patient satisfaction had improved. ${ }^{2,3} \mathrm{~A}$ win-win-win-win. That document includes a copy of a consensus statement signed by 17 of the UK's health care leaders, including the NHS Medical Director and Presidents of the Royal Colleges of Anaesthetists, Physicians, and Surgeons, that concludes:

M. G. Mythen, MD ( $\square)$

Smiths Medical Professor of Anaesthesia and Critical Care, University College London, 1st Floor, Suite A, Maple House, 149 Tottenham Court Road, London W1T 7DN, UK e-mail: m.mythen@ucl.ac.uk

M. G. Mythen, MD

University College London NHS Foundation Trust, London, UK
"We believe that enhanced recovery should now be considered as standard practice for most patients undergoing major surgery across a range of procedures and specialties."

The Enhanced Recovery Partnership Programme in England was started in May 2009 by the Department of Health under the leadership of Professor Sir Mike Richards, the then National Cancer Director, myself (an anesthesiologist/intensivist), and Mr. Alan Horgan, a colorectal surgeon, as National Clinical Leads. ${ }^{1}$ In my view, there is little doubt that major fiscal constraints on public spending had provided us with a burning platform. There was need for urgent change, and enhanced recovery had been identified as a major opportunity for rapid spread and adoption of care pathways that were already being delivered by many teams in the NHS, producing the best results for their patients and, thus, financial savings for the NHS.

Largely influenced by the teachings of Professor Henrik Kehlet, a surgeon from Denmark, enhanced recovery or fast-track was well established in a number of NHS centres, mainly in colorectal and major joint (hip and knee) replacement surgeries, but wider adoption was slow despite vocal enthusiasm. ${ }^{4-8}$ Why? The dominant barriers seemed to be lack of data, inconsistent messaging from health care leaders, and the simple truth that change is hard and time consuming. Sharing good national data was a tipping point. In week one, the leadership team were shown readouts, which were soon to be shared publicly from our national Hospital Episode Statistics (HES), that showed massive inter-hospital variation in length of stay for common coded procedures independent of activity. ${ }^{1}$ A three to fourfold difference in length of stay is hard to explain under any circumstances but even harder when you appreciate that we have a bundled payment system and a single payer (the 
taxpayer via the Treasury and Department of Health). It was clearly understood that increased length of stay can be driven by process or complications, but both are laudable targets. The leadership team subsequently set length of stay targets for eight elective surgical procedures based on the median's that were being reported by the best performing $10 \%$ of institutions at the time. The aims were simply stated that we wanted to see patients helped to get better sooner after surgery, and the results would be judged using our national HES (i.e., despite any inherent warts and all) based on length of stay and readmission rates. We also added patient experience metrics benchmarked against the national annual patient survey. ${ }^{1-3}$

An implementation guide was produced (see above), published on an open source website and sent to all senior health care managers and leaders. ${ }^{1}$ The contents were based on a distillation of best practice that came from around 90 multidisciplinary practitioners who, through a series of workshops, reached consensus and documented key elements of a National Enhanced Recovery Partnership Programme. There are subtle differences for individual surgeries, but the 30 or so major bullet points were generic. The spread and adoption process then started initially through public regional meetings, publications, and a website. The next key step was a direct approach to all chief executives of NHS health care providers to grasp the opportunity to improve the quality of patient care at lower cost. The senior leadership team sent the executives a letter that included the implementation guide and highlighted the value proposition and some estimates of investments they may need to make to achieve far greater returns. The chief executives were encouraged to appoint an internal leadership team that included a champion surgeon, anesthetist, nurse, and manager (administrator). They were also encouraged to give this team a commitment that reasonable requests for investments, as detailed in the implementation guide, would be welcomed. ${ }^{1,9}$

One of my key reflections on the English program is that successful rapid implementation needs champions, but importantly, it also needs unconditional support at the hospital board level, good data, and managers who can see beyond their financial silos and understand that sometimes you have to invest to get returns, not just cut. ${ }^{10,11}$ Change also takes time, and failure to release teams from daily duties to implement change equates to failure to effect change. The early successful adopters in our program were publicly celebrated by the national leadership team and given showcase opportunities at regional and national meetings. Enhanced recovery quickly gained professional consensus and support from the main Royal Colleges and associations. Patients led the production of information that was widely distributed across the NHS, and they have become major drivers of change. ${ }^{1-3,12}$

At a national "summit" in May 2012, the success of the English Enhanced Recovery Partnership Programme was celebrated, and the consensus statement was signed as highlighted above. ${ }^{3}$ Even so, this celebration marked the successful start of a journey and by no means the completion of one. There is still much to be accomplished in our system, and the ambition has recently been documented to include continued progress in the initial four areas, spread to all elective surgeries, and development and implementation in emergency surgery and medical pathways (e.g., pneumonia). ${ }^{3}$

What about evidence? The traditional mantra of "show me the large RCT that proves that enhanced recovery is better blah, blah, blah..." was commonly heard in the first few months but quickly past. As a clinical academic, clinical trialist, and advocate of evidence-based medicine, my natural instinct was also to demand the multicentre randomized controlled trials (RCTs). Nevertheless, I have been convinced that this is not always the best approach to large-scale testing of complex interventions. Instead, implementation science and quality improvement programs underpinned by high-quality data sets are the future of health care innovation such as the spread and adoption of enhanced recovery, which itself can be further underpinned by translational research and RCTs. ${ }^{6,7}$

\section{Diffusion et adoption de la récupération rapide après une chirurgie programmée dans le service national de santé anglais}

Le document Delivering Enhanced Recovery - Helping Patients to Get Better Sooner After Surgery a été publié par le Département britannique du programme de partenariat pour une meilleure guérison (Health Enhanced Recovery Partnership Programme) le 10 mars 2010. ${ }^{1}$ Faisant référence au Service national de santé du Royaume-Uni (le NHS), il est dit dans la préface:

« La récupération rapide transforme les parcours de soins du cancer et des chirurgies programmées dans le NHS en utilisant des interventions basées sur des données probantes comme modèle de soins, ce qui permet aux patients de récupérer plus vite après une chirurgie. Ce guide développé grâce aux apprentissages des centres à travers le Royaume-Uni fournit une base de départ pour soutenir la mise en œuvre de la récupération rapide. »

En novembre 2013, une synthèse des progrès réalisés intitulée Enhanced Recovery Care Pathway a rapporté la réussite d'une mise en œuvre nationale de quatre principaux 
types de chirurgie programmée: colorectale, gynécologique, orthopédique et urologique. ${ }^{2}$ Des milliers d'interventions chirurgicales supplémentaires furent réalisée avec 170000 jours d'hospitalisation en moins. Les durées de séjour cibles avaient été atteintes, les taux de réhospitalisation n'avaient pas augmenté et, enfin, la qualité et la satisfaction des patients avaient été améliorées. ${ }^{2,3}$ Une réalisation quatre fois gagnante. Ce document inclut une copie de l'énoncé de consensus signé par 17 chefs de file en matière de soins de santé au Royaume-Uni, y compris le directeur médical du NHS et les présidents respectifs des collèges royaux des anesthésiologistes, des médecins et des chirurgiens. Ils concluent par ces mots:

«Nous croyons que la récupération rapide doit maintenant devenir la norme pour la majorité des patients subissant une chirurgie majeure dans un large éventail de procédures et de spécialités. »

Le ministère de la santé anglais a commencé le programme de partenariat pour une récupération rapide en mai 2009 sous l'égide du professeur Sir Mike Richards, qui était alors le directeur du centre national du cancer, moi-même (anesthésiologiste/spécialiste de soins intensifs), et du Dr Alan Horgan, chirurgien colorectal, en tant que leaders cliniques au niveau national. ${ }^{1}$ Selon moi, il fait peu de doute que les contraintes fiscales majeures sur les dépenses publiques nous ont fourni un tremplin. Un changement urgent était nécessaire et la récupération rapide avait été identifiée comme offrant une possibilité majeure de diffusion et d'adoption rapides de méthodes thérapeutiques déjà administrées par de nombreuses équipes au sein du NHS, donnant les meilleurs résultats possibles à leurs patients et donc sources d'économies pour le NHS.

Largement influencée par les enseignements du Pr Henrik Kehlet, un chirurgien danois, la récupération rapide ou accélérée était bien installée dans un certain nombre de centres du NHS, principalement pour la chirurgie colorectale et la chirurgie de remplacement des grosses articulations (hanche et genou), mais l'élargissement de son adoption a été plus lent, en dépit de l'enthousiasme manifesté. ${ }^{4-8}$ Pourquoi? Les principaux obstacles semblaient être un manque de données, des messages contradictoires de la part des chefs de file des soins de santé et le simple fait que le changement est difficile et exige du temps pour sa mise en œuvre. Le partage de bonnes données au niveau national a été le moment charnière. Au cours de la première semaine, l'équipe de pilotage a reçu des communiqués qui allaient bientôt devenir publics, de notre service national des Hospital Episode Statistics (HES); ceux-ci montraient une variation interhôpitaux considérable de la durée de séjour pour des procédures couramment codées, indépendantes de l'activité. ${ }^{1}$ Une différence d'un facteur trois ou quatre dans la durée de séjour est difficile à expliquer quelles que soient les circonstances, et encore plus difficile quand vous considérez que nous avons un système de paiement groupé et un payeur unique (le contribuable, par le biais du Trésor et du ministère de la
Santé). Il était clairement compris que l'allongement des durées de séjour pouvait être dû aux procédures suivies ou aux complications, mais il s'agissait là de deux cibles louables. L'équipe de pilotage a ensuite fixé des durées de séjour cibles pour huit procédures chirurgicales programmées basées sur la durée médiane indiquée par les $10 \%$ les plus performants parmi les établissements de soins à cette époque. Les objectifs annoncés étaient simplement que nous voulions voir de l'aide aux patients pour qu'ils récupèrent mieux et plus rapidement après l'intervention chirurgicale et que les résultats seraient jugés au moyen de notre HES national (en dépit de ses défauts inhérents et autres) sur la base de la durée du séjour et des taux de réhospitalisations. Nous avons également ajouté des paramètres de mesure de l'expérience des patients étalonnés à la lumière de l'enquête nationale annuelle auprès des patients. ${ }^{1-3}$

Un guide de mise en œuvre a été produit (voir ci-dessus), publié sur un site Web à source ouverte et envoyé à tous les gestionnaires responsables et chefs de file des soins de santé. ${ }^{1}$ Le contenu reposait sur un distillat des meilleures pratiques provenant d'environ 90 praticiens de disciplines variées qui avaient atteint un consensus au terme d'une série d'ateliers, et documentés les éléments clés d'un programme national de partenariat pour une récupération rapide. Il y a de légères différences individuelles entre les chirurgies mais les plus ou moins 30 points principaux pouvaient s'appliquer à toutes. Le processus de diffusion et d'adoption a d'abord commencé au travers de réunions régionales, de publications et d'un site Web. La principale étape suivante était une approche de tous les directeurs de services de soins du NHS pour saisir la chance d'améliorer la qualité des soins du patient pour un moindre coût. L'équipe de pilotage principale a envoyé une lettre à tous les directeurs qui incluait le guide de mise en œuvre et soulignait la proposition de valeur ainsi que quelques estimations des investissements qu'ils devraient faire pour obtenir des résultats bien supérieurs. Les directeurs étaient encouragés à nommer une équipe de pilotage interne incluant un chirurgien «champion », un anesthésiologiste, un membre du personnel infirmier et un gestionnaire responsable (administrateur). Ils étaient également invités à s'engager auprès de cette équipe à accueillir favorablement les demandes d'investissements, telles que détaillées dans le guide de mise en œuvre. ${ }^{1,9}$

L'une de mes principales réflexions concernant le programme anglais était qu'une mise en œuvre rapide et réussie nécessite des champions et, plus encore, qu'il nécessite un soutien inconditionnel au niveau du conseil d'administration de l'hôpital, de bonnes données et des gestionnaires capables de voir au-delà de leur culture de « silo économique » et de comprendre qu'il est parfois nécessaire d'investissement pour faire des gains, pas simplement de couper dans les budgets. ${ }^{10,11}$ Le changement prend également du temps et ne pas soulager les équipes de leurs tâches 
quotidiennes revient à échouer à mettre le changement en pratique. Ceux qui ont eu du succès rapidement avec l'adoption de notre programme ont été publiquement félicités par l'équipe de pilotage nationale et ont eu la possibilité d'être mis en valeur au cours de réunions régionales et nationales. La récupération rapide a acquis rapidement un consensus professionnel et le soutien de la part des principaux Collèges royaux et associations. Les patients ont guidé la production d'informations largement distribuées dans l'ensemble du NHS et sont devenus les principaux moteurs du changement. ${ }^{1-3,12}$

Le succès du Programme anglais de partenariat pour une récupération rapide a été célébré au cours d'un «sommet» national qui s'est tenu en 2012 et la déclaration de consensus a été signée comme indiqué plus haut. ${ }^{3}$ Toutefois, cette célébration ne faisait que marquer le début d'un voyage réussi et en aucun cas, la fin d'un tel voyage. Il y a encore beaucoup à accomplir dans notre système et cette ambition a été récemment documentée pour $\mathrm{y}$ inclure les progrès continus dans nos quatre domaines initiaux, l'extension à toutes les chirurgies programmées ainsi que son développement et sa mise en œuvre en chirurgie d'urgence et dans des filières médicales (par exemple, la pneumonie). ${ }^{3}$

Qu'en est-il des données probantes? Le mantra traditionnel du «montrez-moi le grand essai clinique randomisé qui prouve que la récupération rapide est meilleure, blablabla... » était souvent entendu au cours des premiers mois, mais a rapidement disparu. En tant qu'universitaire clinicien, investigateur clinique et défenseur de la médecine fondée sur des données probantes, mon instinct naturel exigeait également des essais cliniques randomisés multicentriques. J'ai été néanmoins convaincu qu'il ne s'agissait pas toujours de la meilleure approche pour des tests à grande échelle d'interventions complexes. En revanche, la science de la mise en œuvre et les programmes d'amélioration de la qualité de l'acte soutenus par des ensembles de données de haute qualité constituent le futur de l'innovation dans le domaine des soins de santé, tel que la diffusion et l'adoption de la récupération rapide, qui - elle-même - peut être renforcée par la recherche translationnelle et les essais cliniques randomisés. ${ }^{6,7}$

Potential conflicts Professor Michael Mythen is a professor at UCL and a consultant at UCLH. He has received honoraria for speaking, consultation, and/or travel expenses from Baxter, BBraun, Covidien, Edwards Lifesciences, Fresenius-Kabi, Hospira, and LiDCO. He was National Clinical Lead for the Department of Health Enhanced Recovery Partnership, Smiths Medical Professor of Anaesthesia and Critical Care UCL, and co-inventor of "QUENCH" (pump) IP being exploited by UCL Business. Professor Mythen's institution has also received charitable donations and grants from Smiths Medical Endowment and Deltex Medical. Professor Mythen is also coauthor of the GIFTASUP guidelines on perioperative fluid management, a board member of The Faculty of Intensive Care Medicine, Editor-in-Chief of Perioperative Medicine, on the editorial board of the British Journal of Anaesthesia and the Journal of Critical Care, and a member of the Improving Surgical Outcomes Group. He was a member of the NICE IV fluids guideline development group and Co-Director Xtreme Everest.

Conflits d'intérêts potentiels Le Pr Michael Mythen est professeur à l'UCL et consultant pour l'UCLH. Il a reçu des honoraires comme conférencier, en tant que consultant, et/ou a eu des frais de déplacement pris en charge par Baxter, BBraun, Covidien, Edwards Lifesciences, Fresenius-Kabi, Hospira et LiDCO. Il était le responsable clinique national pour le Département du Health Enhanced Recovery Partnership, Smiths Medical Professeur d'anesthésie et de soins intensifs à l'UCL et co-inventeur $\mathrm{du}$ « QUENCH » (pompe) IP dont l'exploitation est assurée par UCL Business. L'organisme du Pr Mythen a également reçu des dons de bienfaisance et des subventions de Smiths Medical Endowment et Deltex Medical. Le Pr Mythen est également le coauteur des directives GIFTASUP sur la gestion périopératoire des liquides, membre du conseil d'administration de The Faculty of Intensive Care Medicine, Rédacteur en chef de Perioperative Medicine, membre du comité éditorial du British Journal of Anaesthesia et du Journal of Critical Care, ainsi que membre du groupe Improving Surgical Outcomes Group. Il a été membre du groupe ayant élaboré les directives pour la gestion des liquides IV du NICE et co-administrateur de Xtreme EVEREST.

Open Access This article is distributed under the terms of the Creative Commons Attribution Noncommercial License which permits any noncommercial use, distribution, and reproduction in any medium, provided the original author(s) and the source are credited.

\section{References}

1. Enhanced Recovery Partnership Programme. Delivering Enhanced Recovery - Helping Patients to Get Better Sooner After Surgery. March 2010. Available from URL: http:// webarchive.nationalarchives.gov.uk/20130221101407/http://www. dh.gov.uk/prod_consum_dh/groups/dh_digitalassets/documents/ digitalasset/dh_119382.pdf (accessed August 2014).

2. NHS. Enhanced Recovery Care Pathway - a Better Journey for Patients Seven Days a Week and Better Deal for the NHS. Progress review (2012/13) and level of ambition (2014/15). Available from URL: http://www.nhsiq.nhs.uk/8846.aspx (accessed August 2014).

3. NHS. Fulfilling the Potential - a Better Journey for Patients and a Better Deal for the NHS. Available from URL: http://webarchive. nationalarchives.gov.uk/20130221101407/http://www.improvement. nhs.uk/documents/er_better_journey.pdf (accessed August 2014).

4. Knott A, Pathak S, McGrath JS, et al. Consensus views on implementation and measurement of enhanced recovery after surgery in England: Delphi study. BMJ 2012; DOI:10.1136/ bmjopen-2012-001878.

5. Grocott MP, Martin DS, Mythen MG. Enhanced recovery pathways as a way to reduce surgical morbidity. Curr Opin Crit Care 2012; 18: 385-92.

6. Fawcett WJ, Mythen MG, Scott MJ. Enhanced recovery: more than just reducing length of stay? Br J Anaesth 2012; 109: 671-4.

7. Mythen M. Fit for surgery? Anesth Analg 2011; 112: 1002-4.

8. Kehlet $H$, Mythen $M$. Why is the surgical high-risk patient still at risk? Br J Anaesth 2011; 106: 289-91. 
9. Grace C, Kuper M, Weldon S, Lees J, Modasia R, Mythen M. Service redesign. Fitter, faster: improved pathways speed up recovery. Health Serv J 2011; 121: 28-30.

10. NHS. Innovation Health and Wealth, Accelerating Adoption and Diffusion in the NHS. December 2011. Available from URL: http://webarchive.nationalarchives.gov.uk/20130221101407/ http://www.midtech.org.uk/wp-content/uploads/2010/05/Innovation HealthandWealth.pdf (accessed August 2014).
11. Mythen $M G$, Swart M, Acheson $N$, et al. Perioperative fluid management: consensus statement from the enhanced recovery partnership. Perioper Med 2012; 1: 2.

12. NHS. My Role and My Responsibilities in Helping to Improve my Recovery. Steps to Successful Recovery Start Before My Operation. Available from URL: http://www.nhs.uk/Conditions/ enhanced-recovery/Documents/My_Role_My_Responsibilities. pdf (accessed August 2014). 